\title{
Re-coding Black Mirror Chairs' Welcome
}

This volume of proceedings presents the papers from the 2nd edition of the interdisciplinary workshop Re-coding Black Mirror, held on April 24, 2018 in Lyon, France and co-located with The WEB Conference (WWW2018).

Participating to the topical debate of data ethics and algorithmic governance, Re-coding Black Mirror offers the research community tools to reflect on its role in the construction of the technological future and the potential societal implications. The workshop becomes a venue for computer scientists, data scientists and social scientists to create bridges of knowledge.

The complexity of the societal phenomena emerging from the development in web technologies urge for interdisciplinary collaboration. Following the slightly futuristic approach to technology of the British-made sci-fi series Black Mirror, we called scientists to create their dystopic scenarios developed from their own existing technologies. Through this thought experiment, researchers considered potential ethical and social risks of technological advancements offering in some cases possible solutions.

We received eighteen submissions for this workshop, most of which were of high quality and presenting some great insights. Following the reviews of the PC committee, consisted of both social and computer scientists, we accepted ten papers presented in this volume. Review and acceptance statistics are as follows:

\begin{tabular}{|l|c|c|c|}
\hline Submission Type & Reviewed & Accepted & \\
\hline Full Technical Papers & 2 & 2 & $100 \%$ \\
\hline Short Poster Presentations & 16 & 8 & $50 \%$ \\
\hline Total & 18 & 10 & $55 \%$ \\
\hline
\end{tabular}

We are grateful to all PC members of the workshop for their valuable and insightful reviews that gave the authors the possibility to develop their ideas further. A special thank goes to all the authors for their high-quality contributions and the enthusiasm to an emerging and rather alternative workshop.

$\begin{aligned} & \text { Pinelopi Troullinou } \\ & \text { The Open University }\end{aligned}$
Insight@NUIG

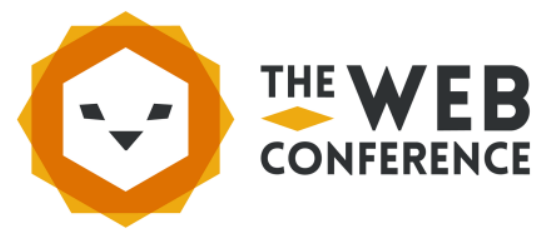




\title{
Re-coding Black Mirror Organization
}

\author{
Organization Committee: Pinelopi Troullinou (The Open University, United Kingdom) \\ Mathieu d'Aquin (Insight @NUIG, Ireland) \\ Ilaria Tiddi (The Open University, United Kingdom) \\ Program Committee: Kirstie Ball (University of St. Andrews, United Kingdom) \\ Valerio Basile (Sapienza University of Rome, Italy) \\ Nikos Bikakis (ATHENA Research Center, Greece) \\ Pompeu Casanovas (Autonomous University of Barcelona, Spain) \\ Sara Degli Esposti (Coventry University, United Kingdom) \\ Stefan Dietze (University of Leibniz, Germany) \\ Seda Guerses (University of Leuven, The Netherlands) \\ Heidi Herzogenrath-Amelung (University of Westminster, United Kingdom) \\ David Lewis (ADAPT Centre, Ireland) \\ Liisa A. Mäkinen (University of Turku, Finland) \\ Andrea Mannocci (The Open University, United Kingdom) \\ Diana Miranda (Keele University, United Kingdom) \\ Giuseppe Rizzo (Politecnico of Turin, Italy) \\ Barry O'Sullivan (University College Cork, Ireland) \\ Nikolas Thomopoulos (University of Green wich, United Kingdom) \\ Daniel Trottier (Erasmus University Rotterdam, The Netherlands) \\ Lachlan Urquhart (University of Nottingham, United Kingdom)
}

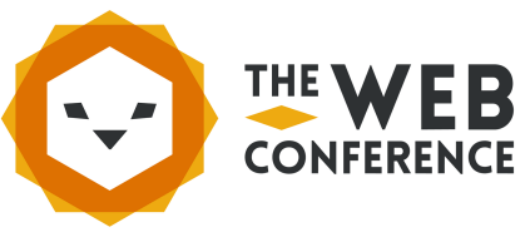

